\title{
Fragmentation of Rest/Activity Patterns in Community-Based Elderly Individuals Predicts Incident Heart Failure
}

This article was published in the following Dove Press journal: Nature and Science of Sleep

\author{
Lei Gao (D) ${ }^{1,2}$ \\ Andrew SP Lim ${ }^{3}$ \\ Patricia M Wong ${ }^{4}$ \\ Arlen $\mathrm{Gaba}^{2}$ \\ Longchang Cui $\mathbb{D}^{2}$ \\ Lei $\mathrm{Yu}^{5}$ \\ Aron S Buchman ${ }^{5}$ \\ David A Bennett ${ }^{5}$ \\ Kun $\mathrm{Hu}$ (D) $^{2,6}$ \\ Peng Li (iD ${ }^{2,6}$ \\ 'Department of Anesthesia, Critical Care \\ and Pain Medicine, Massachusetts \\ General Hospital, Harvard Medical \\ School, Boston, MA 02II4, USA; \\ ${ }^{2}$ Division of Sleep and Circadian \\ Disorders, Brigham and Women's \\ Hospital, Boston, MA 02II5, USA; \\ ${ }^{3}$ Division of Neurology, Department of \\ Medicine, Sunnybrook Health Sciences \\ Centre, University of Toronto, Toronto, \\ ON, USA; ${ }^{4}$ Department of Psychology, \\ University of Pittsburgh, Pittsburgh, PA \\ 15260, USA; ${ }^{5}$ Rush Alzheimer's Disease \\ Center, Rush University Medical Center, \\ Chicago, IL 60612, USA; ${ }^{6}$ Division of \\ Sleep Medicine, Harvard Medical School, \\ Boston, MA 021I5, USA
}

Correspondence: Lei Gao

Department of Anesthesia, Critical Care and Pain Medicine, Massachusetts General Hospital, Harvard Medical School,

Boston, MA 02II4, USA

Tel + I 617 888-294|

Fax + $617732-7337$

Email lgao@mgh.harvard.edu

Peng Li

Division of Sleep and Circadian

Disorders, Brigham and Women's

Hospital, Boston, MA 02115, USA

$\mathrm{Tel}+\mid 617$ 278-006|

Fax + $617732-7337$

Email pli9@bwh.harvard.edu
Study Objectives: Heart failure has previously been linked to sleep disorders that are often associated with frequent disturbances to human rest/activity patterns. We tested whether fragmentation of sustained rest/activity patterns derived from actigraphic recordings at baseline predicts incident heart failure in community-based elderly individuals.

Methods: We studied 1099 community-based elderly adults participating in the Rush Memory and Aging Project who had baseline motor activity monitoring up to 11 days and were followed annually for up to 14 years. Fragmentation was assessed using previously validated indexes, derived from the probability of transitions once sustained rest or activity has been established. Heart failure was recorded via a clinical interview during the annual follow-up. Cox proportional hazards models were constructed to examine the relationship between rest fragmentation index and incident heart failure. Covariates grouped in terms of demographics, lifestyle factors and co-morbidities and cardiovascular risk factors/diseases were included.

Results: Increased rest fragmentation (but not activity fragmentation) was associated with higher risk for incident heart failure. Specifically, a subject with a rest fragmentation at the 90th percentile showed a 57\% increased risk of developing incident heart failure compared to a subject at the 10th percentile in this cohort. This effect was equivalent to that of being over a decade older. These observations were consistent after adjusting for all covariates.

Conclusion: Increased rest fragmentation, a potential surrogate for sleep fragmentation, is independently associated with a higher risk of developing heart failure in community-based elderly adults during up to 14 years of follow-up. Further work is required to examine the specific contributions from daytime napping versus nighttime sleep periods in the elderly, as well as the underlying autonomic and cardio-dynamic pathways that may explain the effects on heart function.

Keywords: sleep fragmentation, heart failure, mobile health, unobtrusive monitoring

\section{Introduction}

Heart failure (HF) remains a common condition in an aging population, with excess morbidity and mortality, incurring significant public health costs. ${ }^{1,2} \mathrm{HF}$ affects an estimated 26 million people worldwide, resulting in more than 1 million hospitalizations annually in both the United States and Europe. ${ }^{2}$ Given that half of those hospitalized patients die within five years, ${ }^{3}$ there is a pressing need to better identify those in the community who are at increased risk of developing HF. 
At the same time, disturbances in rest periods (often during sleep) are common in patients with $\mathrm{HF}^{4,5}$ Mechanisms linking these disturbances and HF include multiple arousals, which can lead to fragmentation during sleep, causing excessive sympathetic activation ${ }^{6}$ and, in turn, moderate the renin-angiotensin-aldosterone system (RAAS) in a maladaptive way. ${ }^{7,8}$ Over time, increased sympathetic activation is accompanied by maladaptive hypertension leading to arteriolar and cardiac wall stress. ${ }^{9-12}$ The cumulative effects from these frequent arousals from sleep, sympathetic surges, higher blood pressures, and repetitive low oxygen levels, compound to adversely suppress the efficiency and performance of the heart muscles, the failure of which is the defining feature of HF.

While there have been strong links between sleepdisordered breathing (SDB) and incident $\mathrm{HF}^{13-15}$ these disorders, including sleep apnea (SA), remain underdiagnosed, and may not fully capture the extent of sub-clinical sleep arousals experienced by elderly adults prior to the diagnosis of HF. ${ }^{14,16}$ There remains a paucity of studies on the longitudinal role of objectively measured sleep function in elderly adults at risk of developing HF. This is due, in part, to reliance on formal clinical diagnoses by sleep specialists, and often requires polysomnography. These are often in short-supply, costly, and time-consuming, thereby limiting the number of community dwellers that can be screened. ${ }^{14}$

Recently, analytical approaches have been successfully implemented with continuous motor activity data to study rest/activity patterns to infer sleep-wake behaviour. ${ }^{17-19}$ Activity monitors can be worn unobtrusively on the wrist without disruption to sleep and normal daily activities in elderly adults in community-based settings. This has the advantage of reaching those who may be medically underserved to capture continuous rest and activity metrics. Given that retired, elderly community dwellers are more likely to have a mixture of rest and activity periods regardless of time of day, we were interested in quantifying the extent to which baseline fragmentation to rest and activity periods affect heart failure risk in future. We analyzed data from 1099 elderly participants from the Rush Memory and Aging Project (MAP). ${ }^{20}$ Subjects were followed for up to 14 years after baseline assessment of rest/activity fragmentation.

\section{Methods}

\section{Study Design}

Data were derived from the MAP, an ongoing prospective, observational cohort study conducted at the Rush Alzheimer's
Disease Center, Rush University Medical Center. ${ }^{20}$ Starting in 1997, subjects were recruited from over forty residential facilities across the metropolitan Chicago area (including subsidized senior housing facilities, retirement communities, retirement homes, social service agencies, and church groups). In 2005, a watch-like device was introduced to record daily movement/motor activity. ${ }^{21}$ Participants were enrolled into detailed annual clinical evaluations that were performed at home to reduce participant burden and enhance follow-up participation. The protocol of the parent MAP study was approved by an Institutional Review Board (IRB) of Rush University Medical Center and conducted in accordance with the Declaration of Helsinki. Written informed consent was obtained, and all participants signed a repository consent to allow their data to be repurposed. More information regarding the data can be found at www.radc.rush.edu. The protocol for this current study was approved by the Partners Healthcare Inc. IRB.

\section{Participants}

Through 2019, 1291 participants without HF had completed baseline rest/activity monitoring. Criteria of exclusion were participants who did not have at least one follow-up visit reporting the HF status $(\mathrm{N}=93)$, or were missing either age information at baseline or HF incidence, precluding an estimation of time to event (total $\mathrm{N}=99$ ). This left 1099 participants for analysis in this study (age at baseline: mean (SD) 80.7 (7.4); range 56-100 years old, female: 837 ).

\section{Data Collection and Preprocessing}

The activity monitor (Actical, Philips Respironics, Bend, OR) was worn on the non-dominant wrist continuously for about 10 days at baseline. Figure 1 shows two typical recordings of motor activity lasting for 7 days used in the study. This device measures acceleration in a direction parallel to the face of the device with a continuous $32 \mathrm{~Hz}$ sampling frequency and integrates into proprietary counts, in 15second epochs (ie, activity data), via a built-in data processor, that reflect the amplitude of movement. ${ }^{22}$

\section{Assessment of Rest and Activity Fragmentation}

The activity recordings were used to quantify fragmentation using previously published methodology. ${ }^{23-25}$ In brief, fragmentation is characterized by probabilities of transitions between binary states of rest and activity. For 


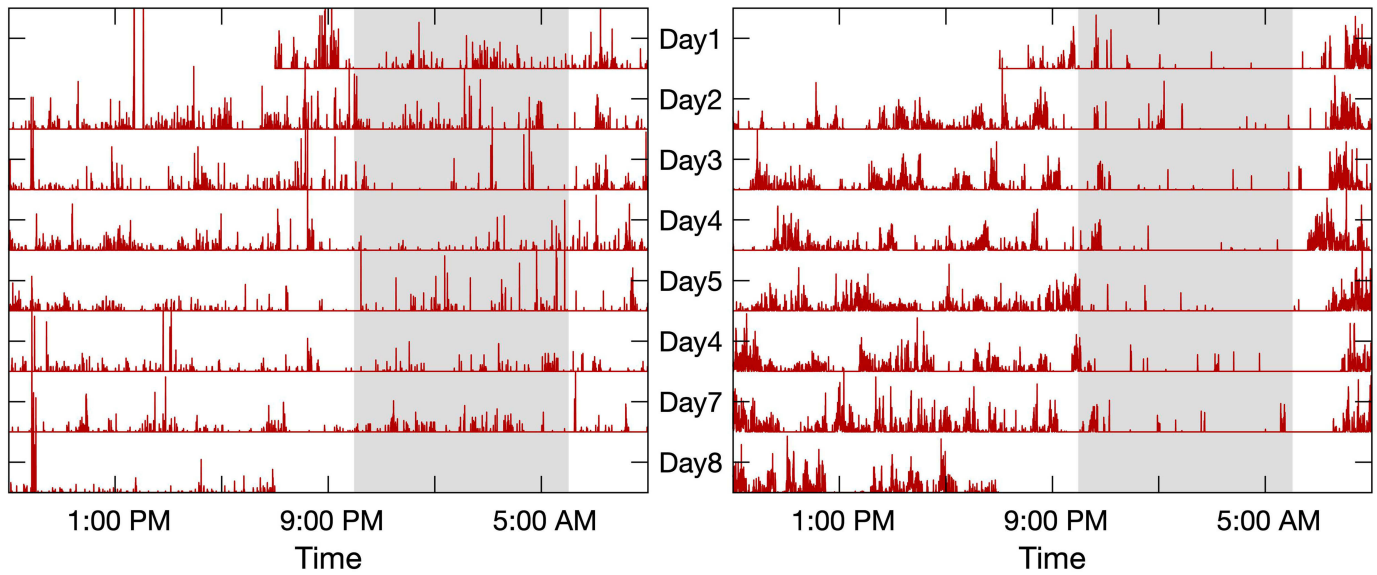

Figure I Rest/activity recordings. The recording on the left panel was from a female subject of $8 \mathrm{I}$ years old with a high sleep fragmentation index $\mathrm{k}_{\mathrm{RA}}$ (3.4\%, the 90 th percentile). This subject developed HF at the third follow-up visit. The recording on the right panel was from a similar female subject of $8 \mathrm{I}$ years old but with a low sleep fragmentation index $\mathrm{k}_{\mathrm{RA}}(2.0 \%$, the 10 th percentile). This subject had also been followed for three years but had not developed HF. The time-axis is on a 24 -hr scale starting at 9:00 AM and ending at 9:00 AM of the following day. Gray-shaded area highlights a common sleep period between 10:00 PM and 6:00 AM.

example, fragmentation index, $\mathrm{k}_{\mathrm{RA}}$ represents a probabilistic state transition from rest-to-active (RA), expressed as a percentage $(0-100) . \mathrm{k}_{\mathrm{RA}}$ is extracted from runs of rest longer than a value $t$, where $t$ is empirically extracted from a given individual's RA probability versus their run duration curve. The $t$ represents the duration at which the RA probability drops to a minimum; for most participants, this was approximately 5 mins. Conversely, $\mathrm{k}_{\mathrm{AR}}$ represents a probabilistic state transition from activeto-rest (AR), also expressed as a percentage (0-100). The higher the indexes the more quickly sustained bouts of rest/activity end in the opposite state and hence the greater the degree of fragmentation. ${ }^{23}$ Of note, indexes were relatively insensitive to choice of thresholds defining activity type (ie, rest vs movement). ${ }^{23}$ Since calculations are based on the entire record, both indexes consider fragmentation irrespective of time of day. However, it has been shown that $\mathrm{k}_{\mathrm{RA}}$ is dominated by periods of greatest inactivity, which are most likely sleep periods, and for the majority of individuals this is at night. ${ }^{23,24}$ Typical patterns of actigraphy recordings with high vs low (90th vs 10th centiles) $\mathrm{k}_{\mathrm{RA}}$ are shown in Figure 1 .

\section{Annual Assessment of Incident Heart} Failure

History of HF is derived from annual clinical interviews. At baseline, the participants are asked: "Have you ever been told by a doctor, nurse, or therapist that you had heart failure?" In each follow-up visit, participants are then asked: "Since your last interview, have you been told by a doctor, nurse, or therapist that you had heart failure?". If so, new incidence was reflected in the dataset starting from that visit onwards.

\section{Assessment of Covariates}

In addition to age, sex, and education years, we grouped covariates in terms of lifestyle factors and co-morbidities [body mass index (BMI), alcohol, cognition, depression, anxiety, insomnia, urinary conditions, and thyroid disease] and finally, cardiovascular risk factors/diseases [smoking, hypertension, diabetes, cholesterol, coronary artery disease (CAD), and claudication]. Age at time of actigraphy assessment was calculated using years elapsed since self-reported date of birth. Education years, BMI, smoking (never/ex/ current), and alcohol use (none/ $>1$ drinks per week) were recorded during the initial interview. We controlled for mean total daily activity to exclude the possibility that any observed associations may result from differences in activity time per day. Mean total daily activity, expressed in thousand counts per day, was calculated from each activity recording by taking the total sum of all activity counts in each recording and dividing by the total number of days. Medications (taken/not taken) were inspected and coded using the Medi-Span system (Medi-Span, Inc.). Presence/ absence of co-morbidities at activity baseline were determined at interview. We coded for presence of urinary conditions [urinary incontinence/spasms, benign prostatic hypertrophy (BPH), or associated medications], which may confound results related to nighttime awakening. Participants were considered to have diabetes, hypertension, or thyroid disease if they were taking medications or 
endorsed a diagnosis on interview. Depressive symptoms were assessed with a 10-item version of the Center for Epidemiologic Studies-Depression Scale and cognition was assessed using a composite score representing global cognition constructed from z-scores of 17 cognitive tests. ${ }^{20}$

\section{Statistical Analysis}

Three Cox proportional hazards models were used to assess the relationship between rest fragmentation index, $\mathrm{k}_{\mathrm{RA}}$ and incident HF - the first occurrence of diagnosis reported by the participants. The initial model controlled for age, sex, education years and total daily activity (Model A). A subsequent model adjusted for lifestyle factors and comorbidities including body mass index (BMI), alcohol, cognition, depression, anxiety, insomnia, urinary conditions, and thyroid disease that may affect both sleep and HF risk (Model B). A full final model included baseline cardiovascular risk/disease variables (Model C). The Efron approximation was applied to account for ties resulting from grouped-survival data as well as scaled Schoenfeld residual plots to confirm the proportional hazards assumption. All statistical analyses were performed using JMP Pro (Ver. 13, SAS Institute, Cary, NC, USA). Statistical significance was determined at an alpha level of 0.05 .

\section{Results}

Clinical characteristics of the 1099 participants included in this study are summarized in Table 1 . The mean age (SD) was 80.7 (7.4); range 56-100 years old. During a mean follow-up of 6.1 years [standard deviation (SD): 3.6, range: 1-14], 80 participants (7.3\% of 1099) developed HF. Rest/activity data were recorded for 9.3 days (SD: 0.9 ) on average (see Figure 1 for typical recordings). The mean fragmentation values for $\mathrm{k}_{\mathrm{RA}}$ and $\mathrm{k}_{\mathrm{AR}}$ were 2.77 (SD: 0.7; range: 1.55-8.24) and 7.26 (SD: 4.0; range: 1.10-44.3), respectively. There was a weak correlation between $\mathrm{k}_{\mathrm{RA}}$ and $\mathrm{k}_{\mathrm{AR}}\left(\mathrm{r}^{2}=0.005, \mathrm{p}=0.015\right)$.

Rest fragmentation, $\mathrm{k}_{\mathrm{RA}}$, was associated with incident HF using a Cox proportional hazards model adjusted for demographics (age, sex and years of education) and physical activity/rest (Table 2; Model A). Specifically, for a 1-SD increase in $\mathrm{k}_{\mathrm{RA}}$, the hazard ratio (HR) of developing HF was 1.26 [95\% confidence interval (CI): 1.06-1.48, $p=0.01$ ]. The effect size is equivalent to that of being 5.2 years older in age. The effect of $\mathrm{k}_{\mathrm{RA}}$ on incident HF did not vary by age or sex ( $p$ 's $>0.05$ for both interaction terms).

To put these results into context, we show model predictions for incident HF for two individuals with high (90th percentile) and low (10th percentile) rest fragmentation
Table I Baseline Characteristics of Study Subjects $(\mathrm{N}=1099)$

\begin{tabular}{|l|l|}
\hline Demographics and physical activity & \\
\hline Age (years) & $80.7(7.4)$ \\
Female (\%) & 76.2 \\
Education (years) & $15.1(3.0)$ \\
Total daily activity (count, $\times 10^{5}$ ) & $16.5(9.7)$ \\
\hline Lifestyle factors and co-morbidities & \\
Body mass index (kg/m $\left.{ }^{2}\right)$ & $27.3(5.3)$ \\
Alcohol, at least I drink per week (\%) & 50.4 \\
Global cognition & $0.08(0.6)$ \\
Depression symptoms & $1.0(1.6)$ \\
Anxiety medication use (\%) & 6.4 \\
Insomnia medication use (\%) & 8.4 \\
Urinary conditions (\%) & 40.6 \\
Thyroid disease (\%) & 30.0 \\
\hline Cardiovascular risk factors/diseases & \\
Smoking (\%) & 40.5 \\
Hypertension (\%) & 64.5 \\
Cholesterol medication use (\%) & 38.5 \\
Diabetes (\%) & 19.2 \\
Coronary artery disease (\%) & 14.1 \\
Claudication (\%) & 8.9 \\
\hline
\end{tabular}

Notes: Data expressed as a count, mean (SD) or percentage (\%). Alcohol (I or more drinks per week), depressive symptoms were assessed with a 10-item version of the Center for Epidemiologic Studies-Depression Scale. Urinary conditions (urinary incontinence/spasms, benign prostatic hypertrophy or diuretic use). Smoking (percentage who are current or ex-smokers). Participants were considered to have diabetes, hypertension, or thyroid disease if they were taking medications or endorsed a diagnosis on interview.

(Figure 2). The participant with rest fragmentation at the 90th percentile showed a $57 \%$ increased risk of developing incident heart failure compared to the participant with a lower rest fragmentation at the 10th percentile; the effect size in this case is equivalent to over a decade increase in age (10.3 years). After adjusting for lifestyle/co-morbidities, we did not find a significant change in the relationship between $\mathrm{k}_{\mathrm{RA}}$ and risk for incident HF (Table 2; Model B). Finally, Model $\mathrm{C}$ adjusted for all available cardiovascular risk factors or diseases; the relationship between rest fragmentation $\mathrm{k}_{\mathrm{RA}}$ and risk for incident HF was weaker but remained significant (Table 2; Model C). For a 1-SD increase in $\mathrm{k}_{\mathrm{RA}}, \mathrm{HR}=1.21$ (95\% CI: $1.02-1.46 ; p=0.03)$. See Supplemental Table S1 for full model results involving $\mathrm{k}_{\mathrm{RA}}$. Note that excluding those scoring lower than 24 out of 30 on MMSE ( $n=97)$, an accepted standard for screening mild cognitive impairment and beyond per the Alzheimer's Association, $\mathrm{k}_{\mathrm{RA}}$ remained predictive $(\mathrm{HR}=1.21,95 \% \mathrm{CI}: 1.01-1.45, \mathrm{p}=0.037)$. No relationship was seen between activity fragmentation $\mathrm{k}_{\mathrm{AR}}$ and HF. 
Table 2 Fragmentation Indexes, $k_{R A}$ (Rest-to-Activity) and $k_{A R}$ (Activity-to-Rest) with Incident Heart Failure

\begin{tabular}{|l|l|l|l|l|l|l|l|}
\hline \multirow{2}{*}{ Model } & \multicolumn{3}{|l|}{$\mathbf{k}_{\mathbf{R A}}$} & \multicolumn{2}{l|}{$\mathbf{k}_{\text {AR }}$} \\
\cline { 3 - 9 } & & $\mathbf{H R}$ & $\mathbf{9 5 \%} \mathbf{C l}$ & $\mathbf{P}$-value & $\mathbf{H R}$ & $\mathbf{9 5 \%} \mathbf{C l}$ & $\mathbf{P}$-value \\
\hline $\mathrm{A}$ & + daily activity & 1.26 & $(1.06$ to 1.48$)$ & 0.01 & 1.07 & $(0.80$ to 1.35$)$ & 0.60 \\
$\mathrm{~B}$ & + lifestyle/co-morbidities & 1.24 & $(1.04$ to 1.48$)$ & 0.01 & 0.88 & $(0.61$ to 1.26$)$ & 0.48 \\
$\mathrm{C}$ & + CVD risk and diseases & 1.21 & $(1.02$ to 1.46$)$ & 0.03 & 0.89 & $(0.61$ to 1.31$)$ & 0.56 \\
\hline
\end{tabular}

Notes: Cox proportional hazards model of baseline measurements. All models included age, sex and education. Model A: HRs (hazard ratios) for incident HF (heart failure) with total daily activity. Model B was adjusted for depression symptoms, global cognition, antianxiety medication use, insomnia medication use, thyroid disease, alcohol ( $\mathrm{I}$ or more drinks per week) and urinary conditions (incontinence/spasms, benign prostatic hypertrophy, diuretic use or associated medications). Model $\mathrm{C}$ was further adjusted for smoking, hypertension, cholesterol medication use, diabetes, coronary artery disease and claudication.

Abbreviations: $\mathrm{HR}$, hazard ratio, $\mathrm{Cl}$, confidence interval.

\section{Discussion}

In this study of 1099 elderly individuals, we present evidence that baseline assessment of a quantitative measure for disruption to rest periods $\left(\mathrm{k}_{\mathrm{RA}}\right)$ is associated with an elevated risk of developing HF during up to 14 years of follow-up; the effect of rest fragmentation could not be accounted for even after extensive consideration for daily activity, chronic medical conditions, medications, nor cardiovascular risk factors.

We previously reported an association between $\mathrm{k}_{\mathrm{RA}}$ and Alzheimer's disease/cognition, ${ }^{25,26}$ but this is the first known link to HF. This may not be surprising given that there is increasing evidence for overlap in shared cardiovascular risk factors in defining optimal brain and heart health, and calls for closer examination by both the American Heart/Stroke associations. ${ }^{27}$ In fact, we recently found links between $\mathrm{k}_{\mathrm{RA}}$

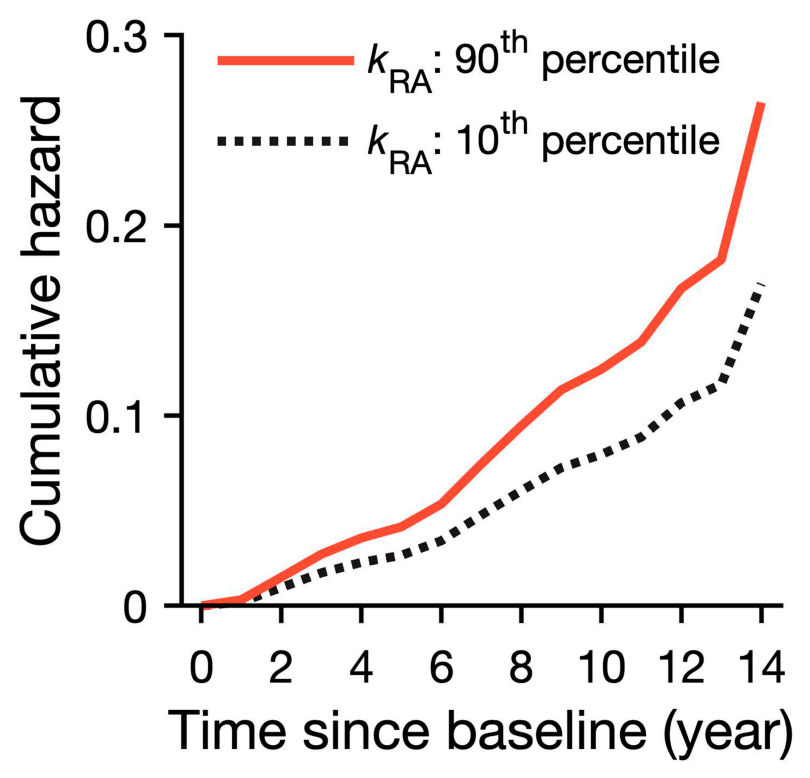

Figure 2 Predicted risk over time for HF from Cox proportional hazards models. The predicted cumulative hazard for incident $\mathrm{HF}$ in two representative participants whose sleep fragmentation were high $\left(k_{R A}=3.4 \%\right.$, the 90th percentile solid line) and low $\left(k_{R A}=2.0 \%\right.$, the 10th percentile dashed line $)$. and cerebral atherosclerosis/infarcts on post-mortem in the same population, ${ }^{28}$ although it remains unclear which proceeded the other (ie, reverse causation), or whether they developed in parallel. However, our longitudinal study design and consideration for extensive covariates make it likely that higher rest fragmentation preceded self-reported $\mathrm{HF}$ in this cohort.

Standard actigraphic metrics such as total daily activity, circadian amplitude, interdaily stability, and intradaily variability were previously shown to correlate with $\mathrm{k}_{\mathrm{AR}} \cdot{ }^{23} \mathrm{We}$ should note that people with poor nighttime sleep may complain about daytime sleepiness or fatigue, both are quite common phenomena in people with $\mathrm{HF}$ and may appear prior to the incidence. ${ }^{5}$ Our observed correlation between $\mathrm{k}_{\mathrm{RA}}$ and $\mathrm{k}_{\mathrm{AR}}$ was weak but may suggest that rest fragmentation is a consequence of fragmented nighttime sleep. For the prediction of $\mathrm{HF}$, the effect of $\mathrm{k}_{\mathrm{RA}}$ remained significant after adjustment for the percentage of rest period while $\mathrm{k}_{\mathrm{AR}}$ had no effect on incident HF. Thus, it is unlikely that $\mathrm{k}_{\mathrm{RA}}$ reflects an effect of lowered daytime activities due to sleepiness or fatigue; rather, it may carry information mainly related to nighttime sleep problems, or attributes of rest/activity organization not captured by published actigraphic measures. ${ }^{23}$ While $\mathrm{k}_{\mathrm{RA}}$ has been shown to be dominated by periods of greatest inactivity, which are most likely sleep periods, and for the majority of individuals this is at night, ${ }^{23,24}$ it is only an estimation of sleep fragmentation per se. This is also calling for more nuanced sleep wearables to be developed that can better distinguish the relationship between sleep, rest period, and risk for HF.

The results also remained significant after controlling for important situational and/or behavioral confounders for $\mathrm{k}_{\mathrm{RA}}$ such as frequent arousals from nocturnal micturition secondary to diuretics, or benign prostatic hypertrophy (Table 2, Model B). The hazard ratio was attenuated after controlling for cardiovascular risks/diseases but remained significant (Table 2, Model C). Potential mechanisms linking $\mathrm{k}_{\mathrm{RA}}$ and HF include the possibility of multiple arousals causing excessive sympathetic activation, ${ }^{6}$ which in turn moderates the 
renin-angiotensin system in a maladaptive way. ${ }^{7,8}$ One key factor is the link between sleep-disordered breathing (SDB) and incident HF. ${ }^{13-15}$ For example, there is a high prevalence of sleep apnea (SA) in individuals with HF. ${ }^{16,29}$ Unfortunately, SA remains underdiagnosed due to a reliance on trained specialists and sleep studies. Reassuringly, the results remain significant after controlling for BMI and presence of high blood pressure, highly prevalent in those with SA.

Despite these results, the exact temporal relationship between HF, SDB and rest fragmentation remains unclear. $\mathrm{k}_{\mathrm{RA}}$ may represent a broader measure of sub-clinical patterns of sleep/rest behavior in elderly community adults, and/or a less advanced form of SDB that may better capture the extent of sleep arousals experienced by elderly adults prior to the diagnosis of HF. ${ }^{14,16}$ Even though our results clearly show that high $\mathrm{k}_{\mathrm{RA}}$ precedes incident HR by many years, it is possible that $\mathrm{k}_{\mathrm{RA}}$ represents sub-clinical heart failure instead, which was unfortunately not assessed at baseline. The extent to which these relationships are causal remains unknown, but to the extent that they are, improvements in sleep may help reduce incidence HF and should be studied in prospective clinical trials.

Strengths of this study include the long-term annual follow-up in a large cohort of community-dwelling adults. Objective, non-intrusive measures of rest fragmentation were obtained over relatively long periods of time (around 10 days) that avoided confounding seen in self-reported sleep measures, minimized the effects of variability from one day to another, and avoided disruption to community dwellers' natural sleep environment, potentially encountered with polysomnography. Furthermore, we were able to adjust for an extensive set of covariates including comorbidities and medications, as well as cardiac risk factors associated with HF.

Amongst several limitations of the current study, this is an observational study that prevents firm conclusions regarding causality of associations found. There may still be unmeasured co-morbidities that theoretically affect both predictor and outcome including SA. The majority of this cohort were elderly women due to the age group and voluntary nature of participation; this limits generalizability to a more general or younger population. Quantitative methods have recently been sought to best characterize physiological data, ${ }^{30-34}$ including sleep behaviour. ${ }^{35,36}$ The challenge has always been to extract meaningful and physiologically relevant insight into the underlying biological trait of interest. ${ }^{37}$ In this case, $\mathrm{k}_{\mathrm{RA}}$ is derived from a model that attempts to approximate true rest/ activity states using detection of transitions. Without proper assessment of sleep using polysomnography, $\mathrm{k}_{\mathrm{RA}}$ remains an approximation of the likelihood of disruption to rest periods. We are cautious to assume that runs of movement are likely to represent wakefulness, and long runs of rest are likely to represent sleep, short runs of rest may represent either quiet wakefulness or light sleep. ${ }^{23-25}$ While others have shown good concordance between actigraphy-derived $\mathrm{k}_{\mathrm{RA}}$ and rest fragmentation derived by polysomnography, ${ }^{25,38}$ much caution is still needed during interpretation. ${ }^{25}$ Future work will involve accurately separating sleep and wake periods, accounting for daytime napping for example, and whether fragmentation is tied to circadian consequences that can partially explain these findings.

There are however, well-documented challenges to this, and in particular the usage of our specific actiwatch (Actical). Specifically, Weiss Allison et al compared PSG with Actical and found a $70 \%$ concordance but with half an hour difference in total sleep time per night for Actical. ${ }^{39}$ Additionally, while valid estimates of steps at moderate and high speeds were shown ${ }^{40,41}$ the Actical may underestimate counts during slower walking, which becomes particularly relevant when discussing studies involving elderly populations in free-living environments. ${ }^{42}$ In theory, this may affect daytime activity fragmentation more than rest fragmentation which is more reliant on a run of activity after sustained inactivity. It remains to be tested whether the seemingly higher threshold of speed of walking needed to register counts can lead to the Actical failing to register more subtle motions during prolonged rest, and thus, artificially lower $\mathrm{k}_{\mathrm{RA}}$. With this potential underestimation for rest fragmentation, effect sizes we found linking $\mathrm{k}_{\mathrm{RA}}$ and HF might be actually smaller than reality. Further work will be needed to untangle these relationships.

One major confounder is sleep apnea (SA), a common medical condition that is highly associated with cardiovascular diseases, including HF. ${ }^{12,29}$ Unfortunately, MAP did not start collecting SA risk scores (Berlin Questionnaire) until 2013. With only 266 MAP participants having SA risk scores, we did not find a link between SA risk and $\mathrm{k}_{\mathrm{RA}}$ ( $p$ > 0.05); MAP has now begun collecting SA data with a separate sleep device. Besides, the reliance on the Berlin questionnaire may not be sufficient to tease out the potential causal chain of subclinical heart failure leading to sleep apnea and rest fragmentation. ${ }^{43}$ For example, others have shown that sleep apnea resulting in heart failure can be both central and obstructive in nature, whereas the Berlin Questionnaire focusses on snoring, a more obstructive sign. ${ }^{43}$

The consensus remains mixed on the causal chain of events. Central SA associated with Cheyne-Stokes respiration 
predicts incident HF; and among patients with HF, it strongly predicts mortality. ${ }^{16,29} \mathrm{SA}$ is also associated with daytime sleepiness and fatigue, likely due to fragmented sleep from recurrent arousals. ${ }^{12}$ Yet, recent studies have been inconclusive as to whether sleep fragmentation itself leads to altered cardiac function. ${ }^{44}$ Perhaps the most accepted pathogenic mechanism linking SA, sleep fragmentation and $\mathrm{HF}$, is the welldocumented occurrence of hypertension over time leading to suppressed cardiac function. ${ }^{12}$ In augmented models, we did control for hypertension and obesity (via BMI), two major factors in SA risk assessments, and the results remained significant. Gradually, the cumulative effects of frequent arousals from sleep, sympathetic surges, cardiac wall stress from higher blood pressures, and low oxygen levels, compound to adversely affect cardiac function. These potentially have intriguing epigenetic consequences resulting from novel molecular pathways. For example, Freitas et al showed that severe obstructive SA was independently associated with microRNAs that have been implicated in heart failure. ${ }^{45}$ Individuals with subclinical heart failure at baseline that were not formally diagnosed using echocardiograms and individuals with undiagnosed sleep apnea could have impacted our findings using the three models. Future data points on such as echocardiography results, biochemistry, such as brain natriuretic peptide, or clinical documentation would strengthen the quality of HF diagnosis. Certainly, future studies are required to better account for SA and its directs effects on clinical markers of suppressed cardiac function leading to HF.

Finally, despite the explicit clinical interviews, as per the design of the MAP study, self-report of HF can be suboptimal as it relies on recall, which can diminish with age. ${ }^{46}$ While we did control for global cognition at baseline, there is likely underreporting of true incidence due to education, degree of involvement in their own care, and poor recall or understanding of clinical terms. The nature of this cohort is such that self-recall by participants is relied upon yearly for all outcomes, medication taken and new medical conditions. Even though nurses are trained to explain the concept of heart failure, misunderstandings are inevitable in such a large cohort. Reassuringly, after excluding those with an MMSE less than $24(\mathrm{n}=97)$, an accepted standard for screening mild cognitive impairment through to severe forms of Alzheimer's dementia per the Alzheimer's Association, kRA remained predictive. In the future, hospital admissions for fluid overload (ie, pulmonary edema) or low cardiac output (ie, cardiogenic shock) would also arguably warrant a formal diagnosis of HF, and we will have the opportunity to improve misclassification using medications and clinical records of HF obtained from Medicare records.

This study presents evidence that rest fragmentation is independently associated with the development of incident HF during up to 14 years of follow-up. Further work is required to examine the underlying autonomic and cardio-dynamic pathways affected by rest fragmentation that may explain their effects on heart function. Ideally, a follow-up study would also acquire baseline quantitative measurements of heart failure via echocardiograms, measurements of baseline sleep apnea indexes with periodic longitudinal follow-ups and definitive heart failure outcomes statistics. This will require collaboration among sleep medicine, cardiology and clinical trial communities to test whether improving general sleep continuity in the elderly community can truly reduce the risk for progression to HF and its associated morbidity and mortality burden.

\section{Acknowledgment}

The authors would like to thank the participants and staff of the Rush Memory and Aging Project and the Rush Alzheimer's Disease Center. This work was supported by NIH grants T32GM007592 (L.G.), R01AG048108, RF1AG059867, RF1 AG064312 (K.H.), R01AG056532 (A.S.B), and R01AG17 917 (D.A.B.).

\section{Disclosure}

The authors report no conflicts of interest in this work.

\section{References}

1. Cook C, Cole G, Asaria P, Jabbour R, Francis DP. The annual global economic burden of heart failure. Int J Cardiol. 2014;171(3):368-376. doi:10.1016/j.ijcard.2013.12.028

2. Ambrosy AP, Fonarow GC, Butler J, et al. The global health and economic burden of hospitalizations for heart failure: lessons learned from hospitalized heart failure registries. $J$ Am Coll Cardiol. 2014;63 (12):1123-1133. doi:10.1016/j.jacc.2013.11.053

3. Loehr LR, Rosamond WD, Chang PP, Folsom AR, Chambless LE. Heart failure incidence and survival (from the Atherosclerosis Risk in Communities study). Am J Cardiol. 2008;101(7):1016-1022. doi:10.1016/j.amjcard.2007.11.061

4. Hastings PC, Vazir A, O'Driscoll DM, Morrell MJ, Simonds AK. Symptom burden of sleep-disordered breathing in mild-to-moderate congestive heart failure patients. Eur Respir J. 2006;27(4):748-755. doi:10.1183/09031936.06.00063005

5. Sharma B, Owens R, Malhotra A. Sleep in congestive heart failure. Med Clin North Am. 2010;94(3):447-464. doi:10.1016/j.mcna.2010.02.009

6. Solin P, Kaye DM, Little PJ, Bergin P, Richardson M, Naughton MT. Impact of sleep apnea on sympathetic nervous system activity in heart failure. Chest. 2003;123(4):1119-1126. doi:10.1378/chest.123.4.1119

7. Mansfield D, Kaye DM, Brunner La Rocca H, et al. Raised sympathetic nerve activity in heart failure and central sleep apnea is due to heart failure severity. Circulation. 2003;107(10):1396-1400. doi:10. 1161/01.CIR.0000056520.17353.4F 
8. Bisogni V, Pengo MF, Maiolino G, Rossi GP. The sympathetic nervous system and catecholamines metabolism in obstructive sleep apnoea. J Thorac Dis. 2016;8(2):243-254. doi:10.3978/j.issn.20721439.2015.11.14

9. Kougias P, Weakley SM, Yao Q, Lin PH, Chen C. Arterial baroreceptors in the management of systemic hypertension. Med Sci Monit. 2010;16(1):RA1-8.

10. Lambert EA, Chatzivlastou K, Schlaich M, Lambert G, Head GA. Morning surge in blood pressure is associated with reactivity of the sympathetic nervous system. Am J Hypertens. 2014;27(6):783-792. doi:10.1093/ajh/hpt273

11. Kario K. Morning surge in blood pressure and cardiovascular risk: evidence and perspectives. Hypertension. 2010;56(5):765-773. doi:10.1161/HYPERTENSIONAHA.110.157149

12. Parish JM, Somers VK. Obstructive sleep apnea and cardiovascular disease. Mayo Clin Proc. 2004;79(8):1036-1046. doi:10.4065/ 79.8.1036

13. Javaheri S, Blackwell T, Ancoli-Israel S, Ensrud KE, Stone KL, Redline S. Sleep-disordered breathing and incident heart failure in older men. Am J Respir Crit Care Med. 2015;193(5):561-568. doi:10.1164/rccm.201503-0536OC

14. Javaheri S, Caref EB, Chen E, Tong KB, Abraham WT. Sleep apnea testing and outcomes in a large cohort of Medicare beneficiaries with newly diagnosed heart failure. Am J Respir Crit Care Med. 2011;183 (4):539-546. doi:10.1164/rccm.201003-0406OC

15. Valika A, Costanzo MR Sleep-disordered breathing during congestive heart failure: to intervene or not to intervene? Journal - SleepDisordered Breathing During Congestive Heart Failure: To Intervene or Not to Intervene?; 2017. Available from: https://www. cfrjournal.com/articles/sleep-disordered-breathing-congestive-hf. Accessed June 13, 2019..

16. Ancoli-Israel S, DuHamel ER, Stepnowsky C, Engler R, Cohen-Zion M, Marler M. The relationship between congestive heart failure, sleep apnea, and mortality in older men. Chest. 2003;124 (4):1400-1405. doi:10.1378/chest.124.4.1400

17. Ancoli-Israel S, Cole R, Alessi C, Chambers M, Moorcroft W, Pollak CP. The role of actigraphy in the study of sleep and circadian rhythms. Sleep. 2003;26(3):342-392. doi:10.1093/sleep/26.3.342

18. Morgenthaler T, Alessi C, Friedman L, et al. Practice parameters for the use of actigraphy in the assessment of sleep and sleep disorders: an update for 2007. Sleep. 2007;30(4):519-529. doi:10.1093/sleep/ 30.4.519

19. Smith MT, McCrae CS, Cheung J, et al. Use of actigraphy for the evaluation of sleep disorders and circadian rhythm sleep-wake disorders: an american academy of sleep medicine clinical practice guideline. J Clin Sleep Med. 2018;14(7):1231-1237. doi:10.5664/ jcsm. 7230

20. Bennett DA, Buchman AS, Boyle PA, Barnes LL, Wilson RS, Schneider JA. Religious orders study and Rush Memory and Aging Project. J Alzheimers Dis. 2018;64(s1):S161-S189. doi:10.3233/ JAD-179939

21. Bennett DA, Schneider JA, Buchman AS, Barnes LL, Boyle PA, Wilson RS. Overview and findings from the Rush Memory and Aging Project. Curr Alzheimer Res. 2012;9(6):646-663. doi:10.2174/156720512801322663

22. Li P, Yu L, Lim ASP, et al. Fractal regulation and incident Alzheimer's disease in elderly individuals. Alzheimers Dement. 2018;14(9):1114-1125. doi:10.1016/j.jalz.2018.03.010

23. Lim ASP, Yu L, Costa MD, et al. Quantification of the fragmentation of rest-activity patterns in elderly individuals using a state transition analysis. Sleep. 2011;34(11):1569-1581. doi:10.5665/sleep.1400

24. Lim ASP, Yu L, Costa MD, et al. Increased fragmentation of rest-activity patterns is associated with a characteristic pattern of cognitive impairment in older individuals. Sleep. 2012;35(5):633640B. doi:10.5665/sleep. 1820
25. Lim ASP, Kowgier M, Yu L, Buchman AS, Bennett DA. Sleep fragmentation and the risk of incident Alzheimer's disease and cognitive decline in older persons. Sleep. 2013;36(7):1027-1032. doi:10.5665/sleep. 2802

26. Lim ASP, Yu L, Costa MD, et al. Increased fragmentation of rest-activity patterns is associated with a characteristic pattern of cognitive impairment in older individuals. Sleep. 2012;35 (5):633-640. doi:10.5665/sleep. 1820

27. Gorelick Philip B, Furie Karen L, Costantino I, et al. Defining optimal brain health in adults: a presidential advisory from the American heart association/american stroke association. Stroke. 2017;48(10):e284-e303. doi:10.1161/STR.0000000000000148

28. Lim ASP, Yu L, Schneider JA, Bennett DA, Buchman AS. Sleep fragmentation, cerebral arteriolosclerosis, and brain infarct pathology in community-dwelling older people. Stroke. 2016;47(2):516-518. doi:10.1161/STROKEAHA.115.011608

29. Drager Luciano F, Doug MR, Ferran B, Geraldo L-F, Susan R. Sleep apnea and cardiovascular disease. Circulation. 2017;136 (19):1840-1850. doi:10.1161/CIRCULATIONAHA.117.029400

30. Li P, Liu C, Li K, Zheng D, Liu C, Hou Y. Assessing the complexity of short-term heartbeat interval series by distribution entropy. Med Biol Eng Comput. 2015;53:77-87. doi:10.1007/s11517-014-1216-0

31. Gao L, Smielewski P, Czosnyka M, Ercole A. Early asymmetric cardio-cerebral causality and outcome after severe traumatic brain injury. J Neurotrauma. 2017;34(19):2743-2752. doi:10.1089/neu.2016.4787

32. Gao L, Smieleweski P, Czosnyka M, Ercole A. Cerebrovascular signal complexity six hours after intensive care unit admission correlates with outcome after severe traumatic brain injury. J Neurotrauma. 2016;33(22):2011-2018. doi:10.1089/neu.2015.4228

33. Yan C, Li P, Liu C, Wang X, Yin C, Yao L. Novel gridded descriptors of poincaré plot for analyzing heartbeat interval time-series. Comput Biol Med. 2019;109:280-289. doi:10.1016/j.compbiomed.2019. 04.015

34. Gao L, Smieleweski P, Li P, Czosnyka M, Ercole A. Signal information prediction of mortality identifies unique patient subsets after severe traumatic brain injury: a decision-tree analysis approach. J Neurotrauma. 2019. doi:10.1089/neu.2019.6631

35. Gao L, Li P, Hu C, et al. Nocturnal heart rate variability moderates the association between sleep-wake regularity and mood in young adults. Sleep. 2019;42:5. doi:10.1093/sleep/zsz034

36. Li P, Morris CJ, Patxot M, et al. Reduced tolerance to night shift in chronic shift workers: insight from fractal regulation. Sleep. 2017;40:7. doi:10.1093/sleep/zsx092

37. Li P, Lim ASP, Gao L, et al. More random motor activity fluctuations predict incident frailty, disability, and mortality. Sci Transl Med. 2019;11(498):516. doi:10.1126/scitranslmed.aax1977

38. Cole RJ, Kripke DF, Gruen W, Mullaney DJ, Gillin JC. Automatic sleep/wake identification from wrist activity. Sleep. 1992;15 (5):461-469. doi:10.1093/sleep/15.5.461

39. Weiss Allison R, Johnson Nathan L, Berger Nathan A, Susan R. Validity of activity-based devices to estimate sleep. J Clin Sleep Med. 2010;06(04):336-342. doi:10.5664/jcsm.27874

40. Feito Y, Bassett DR, Thompson DL. Evaluation of activity monitors in controlled and free-living environments. Med Sci Sports Exerc. 2012;44(4):733-741. doi:10.1249/MSS.0b013e3182351913

41. Johnson M, Meltz K, Hart K, Schmudlach M, Clarkson L, Borman K. Validity of the Actical activity monitor for assessing steps and energy expenditure during walking. J Sports Sci. 2015;33(8):769-776. doi:10.1080/02640414.2014.964747

42. Johnson M. Activity monitors step count accuracy in community-Dwelling older adults. Gerontol Geriatric Med. 2015. doi: $10.1177 / 2333721415601303$

43. Netzer NC, Stoohs RA, Netzer CM, Clark K, Strohl KP. Using the Berlin Questionnaire to identify patients at risk for the sleep apnea syndrome. Ann Intern Med. 1999;131(7):485. doi:10.7326/00034819-131-7-199910050-00002 
44. Cabrera-Aguilera I, Benito B, Tajes M, et al. Chronic sleep fragmentation mimicking sleep apnea does not worsen left-ventricular function in healthy and heart failure mice. Front Neurol. 2019;10:1364. doi:10.3389/fneur.2019.01364

45. Freitas LS, Silveira AC, Martins FC, et al. Severe obstructive sleep apnea is associated with circulating microRNAs related to heart failure, myocardial ischemia, and cancer proliferation. Sleep Breath. 2020. doi:10.1007/s11325-019-02003-1
46. Danckert SL, Craik FIM. Does aging affect recall more than recognition memory? Psychol Aging. 2013;28(4):902-909. doi:10.1037/ a0033263

\section{Publish your work in this journal}

Nature and Science of Sleep is an international, peer-reviewed, open access journal covering all aspects of sleep science and sleep medicine, including the neurophysiology and functions of sleep, the genetics of sleep, sleep and society, biological rhythms, dreaming, sleep disorders and therapy, and strategies to optimize healthy sleep.

Submit your manuscript here: https://www.dovepress.com/nature-and-science-of-sleep-journa
The manuscript management system is completely online and includes a very quick and fair peer-review system, which is all easy to use. Visit http://www.dovepress.com/testimonials.php to read real quotes from published authors. 